\title{
The signaling involved in autophagy machinery in keratinocytes and therapeutic approaches for skin diseases
}

\author{
Li Li ${ }^{1}$, Xu Chen ${ }^{1, *}$ and Heng Gu${ }^{1, *}$ \\ ${ }^{1}$ Institute of Dermatology, Chinese Academy of Medical Science \& Peking Union Medical College, Jiangsu Key Laboratory of \\ Molecular Biology for Skin Diseases and STIs, Nanjing, China \\ * These authors have contributed equally to this work \\ Correspondence to: Heng Gu, email: doctor_guheng@hotmail.com \\ Xu Chen, email: doctor_chx@hotmail.com \\ Keywords: autophagy, keratinocyte, skin, skin disease, autophagy-related gene \\ Received: January 16, $2016 \quad$ Accepted: April 26, $2016 \quad$ Published: May 12, 2016
}

\section{ABSTRACT}

Autophagy is responsible for the lysosomal degradation of proteins, organelles, microorganisms and exogenous particles. Epidermis primarily consists of keratinocytes which functions as an extremely important barrier. Investigation on autophagy in keratinocytes has been continuously renewing, but is not so systematic due to the complexity of the autophagy machinery. Here we reviewed recent studies on the autophagy in keratinocyte with a focus on interplay between autophagy machinery and keratinocytes biology, and novel autophagy regulators identified in keratinocytes. In this review, we discussed the roles of autophagy in apoptosis, differentiation, immune response, survival and melanin metabolism, trying to reveal the possible involvement of autophagy in skin aging, skin disorders and skin color formation. Since autophagy routinely plays a double-edged sword role in various conditions, its functions in skin homeostasis and potential application as a therapeutic target for skin diseases remains to be clarified. Furthermore, more investigations are needed on optimizing designed strategies to inhibit or enhance autophagy for clinical efficacy.

\section{OVERVIEW OF AUTOPHAGY AND AUTOPHAGY MACHINERY}

Autophagy is a conserved catabolic process in which cellular constituents including proteins, organelles and invaded microorganisms are captured and targeted for lysosomal degradation. The process is initiated by generation of the double-membrane autophagosomes which then fuse with lysosomes to form autolysosomes where all contents are enzymatically digested. Autophagy is involved in a large range of cell physiological processes, including immune response, tumorigenesis, differentiation, apoptosis, anti-microbial defense etc. [1-3]. Disordered autophagy has been reported to be associated with a wide range of human diseases such as neurodegenerative diseases, cardiomyopathies, infectious diseases and cancers, and autophagy modulation has been already considered as a potential target for therapy of these diseases $[4,5]$. Activation of autophagy is characterized by specific intracellular biochemical changes. Nearly forty autophagy-related (ATG) proteins have already been identified to participate in different steps of autophagy process, including the recognition of target cargo, and formation, migration, fusion and maturation of autophagosomes [6-8].

Mammalian target of rapamycin (mTOR) is the key up-stream regulator of autophagy, and reduction of mTOR activity is usually accompanied by enhanced phosphorylation of AMP Kinase (AMPK) under metabolic stresses. AMPK, a key energy sensor, can promote autophagy through interacting with tuberous sclerosis complex 2 (TSC2) heterodimer, mTOR complex 1 (mTORC1) subunit raptor or UNC-51-like kinase 1 (ULK1) (homolog of yeast ATG1) [9-11]. Two upstream kinases, Liver kinase B1 (LKB1) and calcium/calmodulindependent protein kinase (CaMK) are responsible for activating AMPK $[12,13]$. Lower energy state could trigger AMPK phosphorylation and subsequent inhibited mTORC1 accompanied with dephosphorylation of p70 S6 kinase (p70S6K) [14, 15].

Two ubiquitin-like conjugation systems of ATG5ATG12 protein complex and microtubule-associated 
protein light chain 3 (LC3) are essential for the process of autophagosome formation. ATG5 conjugates with ATG12, an ubiquitin-like molecule, by a series of ubiquitinationlike reactions involving ATG7 and ATG10 [16]. ATG5ATG12 then conjugates with ATG16L forming ATG5ATG12-ATG16L1 complex which is essential for the initiation of phagophore elongation [17]. LC3 is a specific component of autophagosome membrane. Precursor of LC3 protein synthesized in the cytoplasm is converted to LC3-I form through cleaving carboxy terminus by cysteine protease ATG4, and then its glycine residue is exposed for E1-like enzyme ATG7. Finally, LC3-I is converted to LC3II through conjugating with phosphatidylethanolamine (PE) under catalysis of ATG7 and ATG3 [18]. Notably, ATG7 is a key regulator for autophagosome formation and one of the most important ATG members, based on which many animal models are designed and established to investigate the role of autophagy in regulating cell physiological processes. The Rac1-Armus-Rab7 axis was recently reported to participate in regulating recruitment of LC3 to autophagosomes in keratinocytes [19]. TBC/ RabGAP Armus, especially inactivates Rab7, is an effector of Rac1, a small GTPase involving in a wide range of cellular function regulation including cell adhesion, migration, survival and mitosis and cytokinesis, etc. [20-22]. Inactivated Armus delays autophagy flux by blocking the initiation of phagophore, while Rab7 is transiently activated. Activated Rac1 competes with LC3 for Armus to prevent its recruitment to autophagosomes. Intracellular components targeted for autophagy-related degradation are sequestered and bound to LC3 by adaptor proteins like p62/SQSTM1, neighbor of BRCA1 gene 1 (NBR1) protein and autophagy-linked FYVE protein (ALFY) [23-25]. Besides, Beclin1, a component of Class III phosphatidylinositol 3-kinase (PtdIns3K) complex, is another key modulator that must be mentioned. Beclin1 and PtdIns3K are collaboratively involved in the onset of autophagy [26]. It is important to be aware that Beclin1 is inhibited through its bindings with the apoptosis inhibitor B-cell lymphoma protein $2(\mathrm{Bcl} 2)$ and caspase-mediated cleavage can inactivate Beclin1-induced autophagy, suggesting the involvement in the crosstalk between apoptosis and autophagy [26-29].

Besides the canonical autophagy pathway, the ATG5/ATG7-independent alternative process of autophagy was also described in keratinocytes [30]. ATG7 blocking in mouse models has no functional consequence for skin development, suggesting that it might be not workable to impair autophagy machinery by simply inhibiting one autophagy-related gene, or there might be an alternative pathway [31]. The non-canonical autophagy pathway with the characteristic of Rab9-positive double-membrane vesicles, which is ATG5/ATG7- or LC3- independent but Beclin1-dependent, was observed in keratinocytes, and a major block or impairment of canonical autophagy pathway has no effect on the expression of Rab9 [30].
The canonical and non-canonical autophagy pathway in keratinocytes with molecule effectors repressing or promoting autophagy process which would be introduced later were described in Figure 1.

\section{AUTOPHAGY AND KERATINOCYTE BIOLOGY AND PATHOLOGY}

Epidermis mainly consists of multilayered continuously renewing keratinocytes, and this structure forms the epidermal barrier which necessarily contributes to the defensive responses against various environmental stimulators, such as heat, cold, trauma, radiation and infection. Increasing findings indicate that autophagy plays an important role on keratinocyte biology and pathology, and involves in keratinocyte-related cutaneous disorders, including psoriasis, zoster herpes and cutaneous squamous cell carcinoma (SCC) [32-35].

\section{Role of autophagy in keratinocyte differentiation}

Differentiation is considered as a special way to death for keratinocytes and essential for the maintenance of tissue homoeostasis. The terminal differentiation in keratinocytes is distinctive and usually named as keratinization. The markers of differentiation such as expression of keratin and involucrin and decrease of $\beta 1$ integrin appear in different steps of the keratinization [27]. Sequential differentiation or keratinization is accompanied by the degradation of intracellular constituents and activation of lysosomal enzymes [36]. The differentiationspecific expression and localization of LC3 exhibit in keratinocytes [37]. Series of studies based on autophagy inhibition confirmed the role of autophagy in epidermal keratinization process.

Histochemically, the ATG7-deficient mice with low autophagy level showed reduction in diameter and number of keratohyalin and trichohyalin granules, decreased filaggrin, thickened outer root sheath, acanthosis and hyperkeratosis, suggesting that autophagy in keratinocytes participates in differentiation of epidermis [38]. In further, keratinocytes treated with 3-methyl-adenine (3-MA), an inhibitor of autophagy, or of ATG5-depletion showed impairment of differentiation [30]. 3-MA could also block the reversal process of differentiation caused by metabolic stress-induced autophagy [27]. However, the ATG7-deficient mice are resistant to dye penetration into skin and normal transepidermal water loss, indicating that the constitutively active autophagy in epidermis is not essential for skin barrier function [31].

Several proteins were reported recently to involve in regulation of keratinocyte differentiation targeting on distinct autophagy machinery effectors. Investigation on these proteins contributes to integrate autophagy signaling controlling differentiation, even though some 


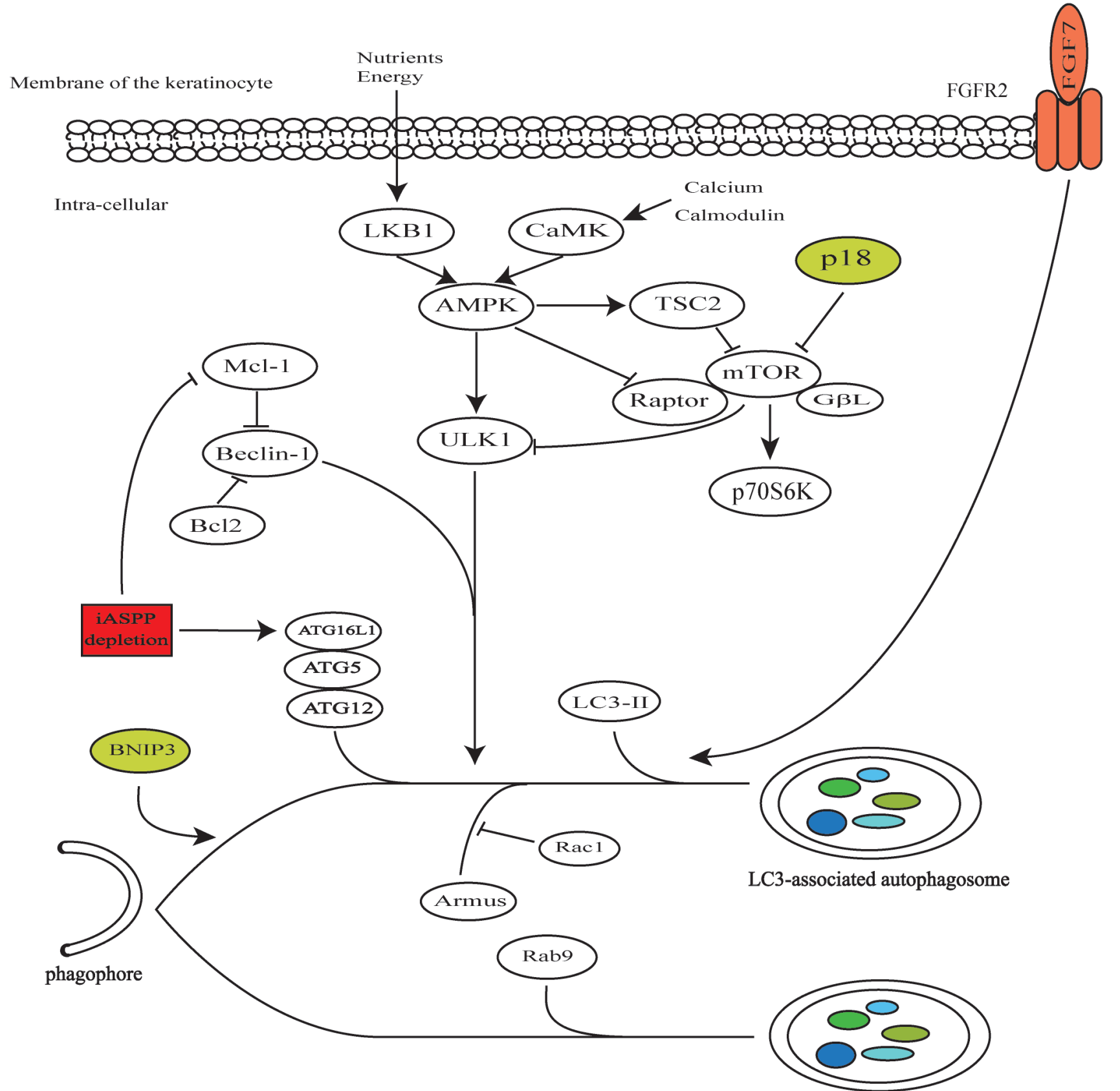

Rab9-associated autophagosome

$\rightarrow$ Induce

$\longrightarrow$ Inhibit

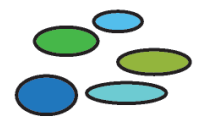

Cargoes of autophagy

Figure 1: Canonical and non-canonical autophagy pathway in keratinocytes. In response to changes of nutrients, energy and intracellular calcium and calmodulin, LKB1 and CaMK activate AMPK following direct modulation of downstream TSC2, raptor and ULK1. The mTOR inhibited by TSC2 or p18 is accompanied with inhibited p70S6K. Beclin1 promoting autophagy process while is inhibited through its bindings with Bcl2 or Mcl-1. ATG5-ATG12-ATG16L1 complex and LC3-II engage in the process from initiation of phagophore elongation to autophagosome formation. The iASPP depletion represses Mcl-1 or promotes ATG5-ATG12-ATG16L1 action. Armus promotes initiation of phagophore which could be blocked by Rac1. Rab9 could be assembled to double-membrane vesicles independently of ATG5/ATG7 and LC3. FGF7-FGFR2 signal stimulates the processes of formation and turnover of autophagosomes. BNIP3 promotes autophagy process but without recognized molecular target yet. 
of these proteins have not been revealed for recognized molecular targets. Fibroblast growth factor $7 /$ keratinocyte growth factor (FGF7/KGF) induced autophagy in human keratinocytes via phosphatidylinositol 3-kinase (PI3K)Akt-mTOR pathway, which was proved by the evidence that autophagy depletion counteracted the FGF7-enhanced increase of early keratinocyte differentiation [39]. The mTORC 1 is speculated the target of $\mathrm{p} 18$ protein, a novel membrane adaptor. The p18-depleted epidermis exhibits severe defects on stratum corneum development and corneocyte formation, and these immature corneocytelike cells displayed lots of autophagosomes [40]. Normal keratinocytes are of high autophagic activity, and the autophagosomes are rapidly degraded by fusion with lysosomes. Analyses of p18-ablated keratinocytes in the presence of bafilomycin (a V-ATPase inhibitor interrupting autophagosome-lysosome fusion step) revealed that the p18-related complex is required for the formation of autolysosomes by facilitating lysosomal fusion. Furthermore, considering the involvement of p18 in the activation of mTORC1, and the target of p18 is lysosome function, a description on $\mathrm{p} 18-\mathrm{mTORC} 1$ signal function is almost certain [41].

The iASPP protein, a member of ASPP (apoptosis stimulating protein of p53) family, negatively modulates function of $\mathrm{p} 53$. The potential link between the iASPPfacilitated autophagy and differentiation in keratinocytes was revealed. Down-regulation of iASPP increased the metabolic form of autophagy against cell death with according changes of a series of autophagic markers including mTORC1, ULK1, Beclin1, p62 and LC3. Furthermore, keratinocytes silenced for iASPP generated thicker epidermis [30]. In the basal layer of epidermis, ATG5-ATG12 partially colocalizes with iASPP which physiologically represses the interaction of ATG5-ATG12 with ATG16L1. Additionally, Bcl2 and adenovirus E1B 19-kDa-interacting protein 3 (BNIP3), an atypical proapoptotic $\mathrm{BH} 3$-only protein, was described to promote differentiation of keratinocytes by inducing autophagy machinery [42].

\section{Role of autophagy in melanin metabolism}

Epidermal melanin determines the skin color which is associated with ethnic diversity. The melanosomes originated in melanocytes are the main organelles in charge of the melanin biosynthesis. The mature pigmented melanosomes then are transferred into neighboring keratinocytes. Actually, the rates of melanosomes transfer and degradation in keratinocytes determine skin color rather than the melanosome biosynthesis in melanocytes $[43,44]$. The autophagy in keratinocytes is the primary mechanism contributing to the skin color development by regulating melanosome degradation. Besides the conventional function of removing the defective melanosomes from melanocytes, autophagy protects cells from melanosome-generated toxicity through eliminating melanosomes in keratinocytes [43, 44]. For instance, it was observed that melanosomes were engulfed into phagosomes in the keratinocytes of the lesion of hypomelanosis [44]. As the distribution patterns of the melanosomes in keratinocytes partly determine skin color, autophagy may play a prominent role in hypomelanosis. Modulating the autophagy machinery might be a better strategy than the melanin synthesis or transfer for treating skin pigmentation disorders or skin cosmetology.

It was reported that FGF7-FGF7 receptor (KGFR/ FGFR2) signal involves in skin color regulation, considering that FGFR2 is more expressed in the light keratinocytes (cells derived from light skins) and melanosome uptake is in a FGF7-dependent way [45, 46]. It is interesting that the FGF7-FGFR2 signal is able to promote the autophagy process through stimulating the formation and turnover of autophagosomes [39]. In addition, ultraviolet (UV) B-induced persistence of melanosomes in keratinocytes could be due to the eased autophagy after internalization and degradation of FGFR2 [47]. Figure 2 describes the transferring event of melanosomes originated in melanocytes to neighboring keratinocytes and relevant signaling involving in the activation of autophagy machinery in charge of melanosome degradation in keratinocytes.

\section{Role of autophagy in immune response}

As the accumulation of damaged intracellular organelles like mitochondria and invasive pathogens can cause inflammation, it is not surprising that autophagy functions serves as an anti-inflammatory machinery by eliminating dysfunctional organelles, microbes and viruses, and participates in immune response. For example, overexpression of some inflammatory cytokines, cathelicidin/LL-37 and S100A7 (psoriasin) is related to inhibition of autophagy $[48,49]$.

Toll-like receptors (TLRs), a group of key recognition molecules in immunity system, involve in the production of antimicrobial immune and pro-inflammatory mediators by activating downstream signals like nuclear factor $\kappa \mathrm{B}(\mathrm{NF}-\kappa \mathrm{B})$ and extracellular regulated protein kinase 1/2 (Erk1/2). A number of TLRs are constitutively expressed or inducible in keratinocytes, and the overexpression of some TLRs is related with chronic cutaneous inflammatory diseases such as atopic dermatitis, psoriasis and acne vulgaris [50, 51]. It was reported that TLRs can mediate the cross-link between autophagy and immune signaling in keratinocytes. For example, the activated TLR2/6 led to an increase of p62 expression and autophagy level, and some components in TLR/NF-кB signal such as myeloid differentiation primary response protein 88 (MyD88) and TNFR-associated factor 6 (TRAF6) were necessary for this induction effect [48]. The scaffold protein p62 involves in triggering TLR-induced 
inflammation as the down-regulation of p62 reduced the activity of NF- $\kappa \mathrm{B}$ promoter and expression of cathelicidin. Mediator role of autophagy machinery in transferring TLR signal to the inflammation pathway effector was described in Figure 3. Considering that expression of p62 and TLRs was significantly increased in psoriatic skin, it is highly possible that autophagy participates in this disease and many other cutaneous disorders associated with inflammations.

Furthermore, activation of autophagy machinery in immune response is meaningful to mitochondrial homeostasis. S100A7 (psoriasin) is distributed in the cytoplasm of normal and terminally differentiated keratinocytes, and plays an important role in the cell surface defense for its antimicrobial activity. The overexpression of S100A7 is related to some epidermal inflammatory diseases and skin cancers [52]. The over- expression of S100A7 reversed the increase of Beclin1 and LC3B expression in the presence of LPS in keratinocytes, suggesting that the inhibitory effect of S100A7 on autophagy might be somewhat relevant to the accumulation of damaged mitochondria under lipopolysaccharide (LPS) treatment, as S100A7 is important for mitochondrial biogenesis [49]. Thus moderating keratinocyte autophagy to reduce inflammation may have important implications for the development of novel therapeutic strategies to improve inflammatory disbalance in psoriasis therapy.

\section{Role of autophagy in antimicrobial defense}

As we discussed above, autophagy is regarded as the mechanism to remove damaged organelles and recycle nutrients. It is now well accepted that the keratinocytes

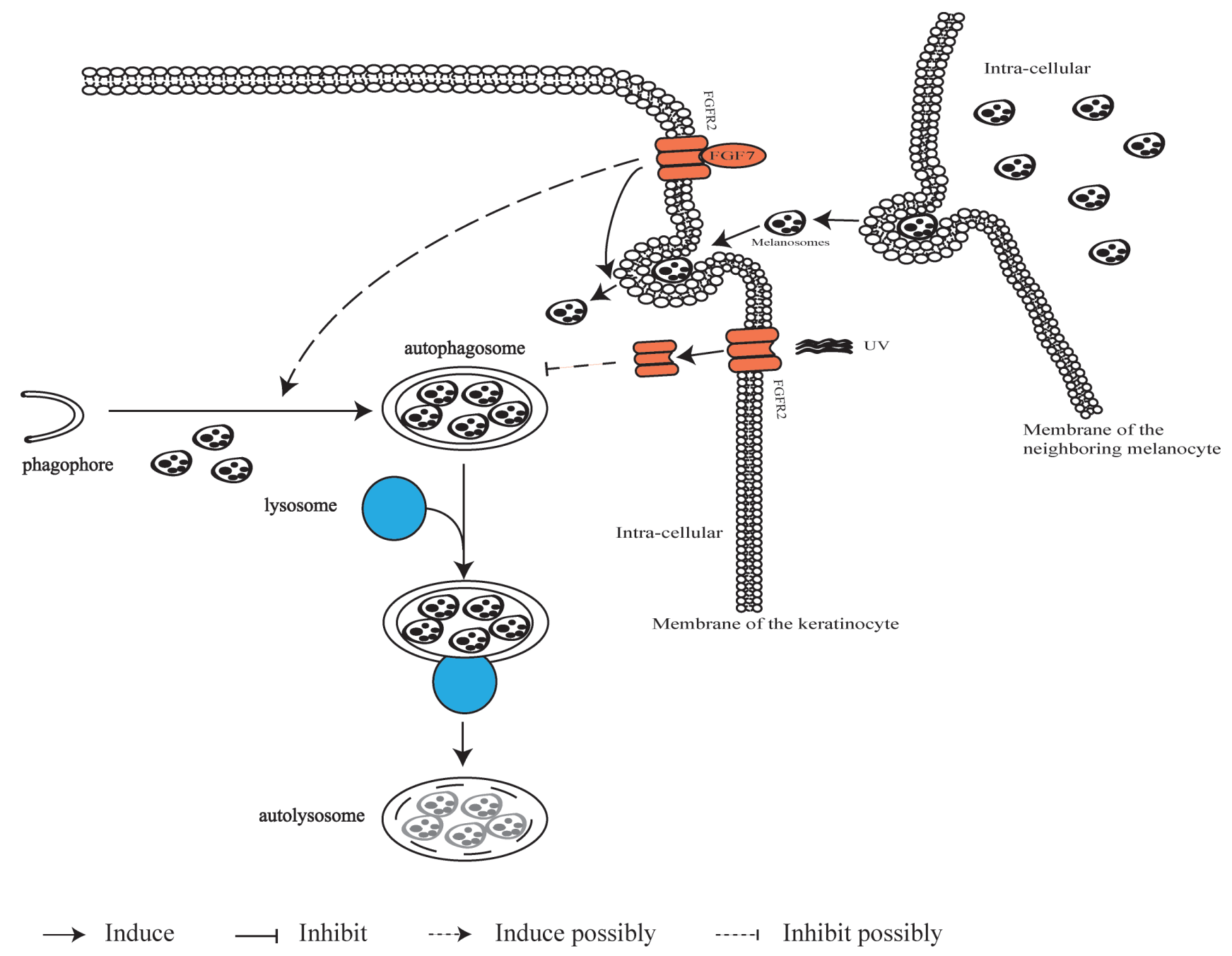

Figure 2: Autophagy machinery in melanin metabolism event. Pigmented melanosomes originated in melanocytes are transferred into neighboring keratinocytes, and the entering process is related to activation of FGF7-FGFR2 signal. UV radiation promotes FGFR2 internalization, and subsequent degradation of FGFR2 by autophagosomes/autolysosomes would ease autophagy capacity to degrade melanosomes. 
serve as the primary component cells of epidermal barrier, and autophagy in keratinocytes involves in host defense against exogenous pathogens and contributes to the innate immune defense by eliminating intracellular viruses and microbes. In contrary, activated autophagy can also be "deceived" by the mutants of some microbes to evade the activation of caspase- 1 and inflammasome, and these mutants resist the keratinocyte-mediated clearance [53].

Human papillomaviruses (HPVs) are non-enveloped double-stranded DNA viruses preferably infecting mucosal and cutaneous tissues with keratinocytes as the main targets. HPV infection is responsible for the anogenital cancer and parts of oropharyngeal SCC. HPV16 is the most prevalent pathogenic one among over 100 genotypes of HPVs, of which the infectivity differs among cell kinds, even among different keratinocytes [54]. In the
HPV16-infected keratinocytes, autophagy functions as the host defense mechanism to inhibit infection but also plays the part of "accomplice" for cell death. Firstly, considering the importance of Rab5 in the biogenesis and coordination of autophagosomes, HPV16 virions may pass through autophagosomes during entry into the host which is proved by the colocalization of the HPV 16 virions with Rab5-containing compartments [55]. Secondly, autophagy inhibited by 3-MA or ATG7-knockdown could significantly enhance the infectivity of HPV16 and delayed the degradation of HPV16 capsid proteins [54, 130]. The degradation is not completely blocked by autophagy inhibition, thus the alternative routes for the trafficking of HPV16 in the host cytoplasm cannot be excluded. Finally, if the persistent autophagy exceeds a threshold, the cell death would occur. In keratinocytes, HPV16 E7

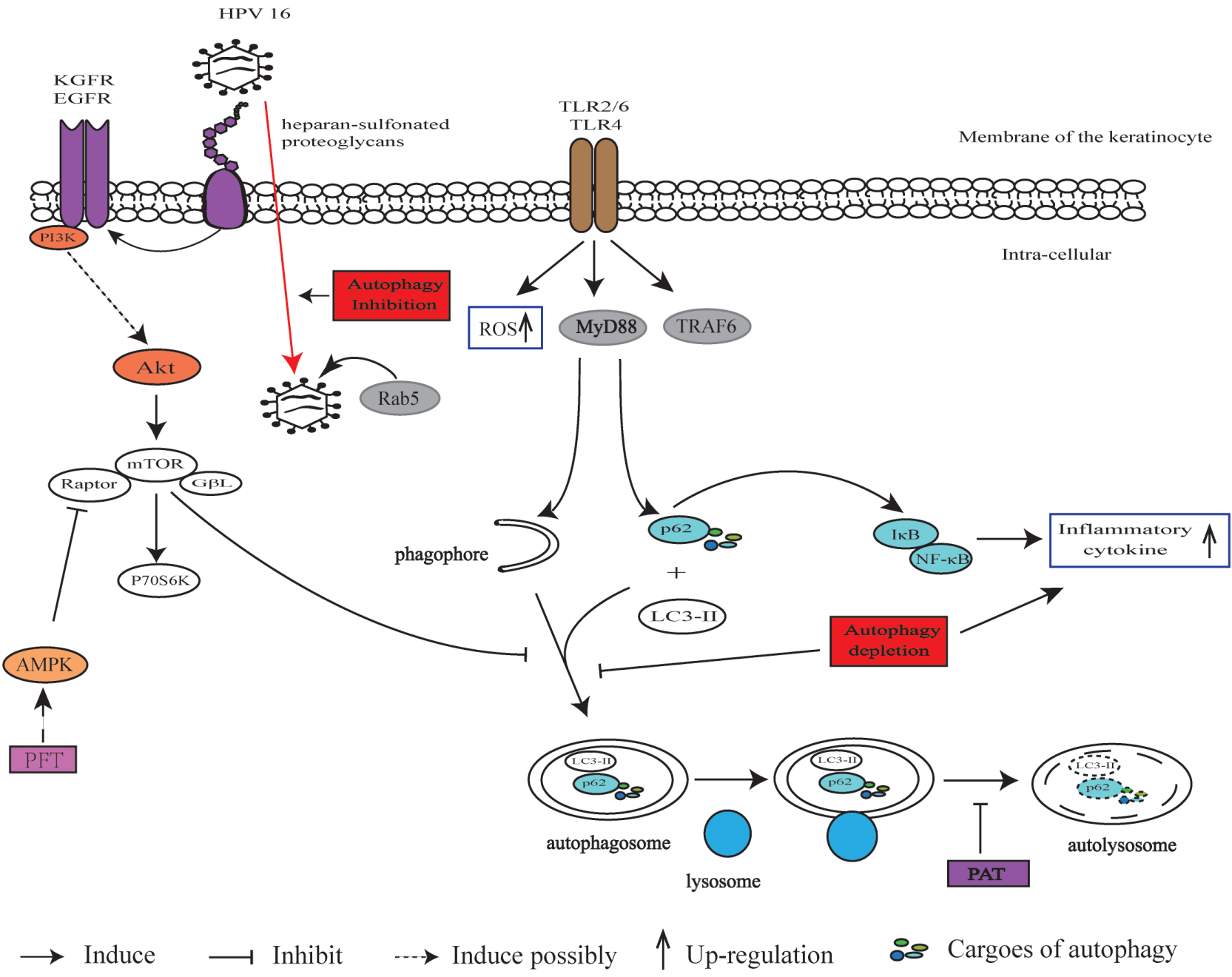

Figure 3: Autophagy machinery in immune response in keratinocytes. Activated TLR2/6 and TLR4 led to increased p62 and activated autophagy through generation of ROS, MyD88 and TRAF6. The p62 promotes inflammatory cytokine generation via NF- $\mathrm{BB}$. EGFR or KGFR is in charge of sensing stimulation from HPVs. HPV virus bind to heparan-sulfonated proteoglycans of cell surface and move to EGFR or KGFR following activation of downstream PI3K/Akt/mTOR pathway. During transport HPVs undergo Rab5 association. PFT induces autophagy with a possible target of AMPK. PAT treatment inhibits autophagosome degradation. 
oncoprotein expression primarily mediates an increase of energy requirements due to aberrant cell proliferation or metabolic stress, which leads to the prolonged autophagy process and the cell death under conflicting growth signals [56]. Suppressed autophagy is necessary for the infected cells to survive, which is proved by the experiment of depleting viral early genes [57].

Since the tight involvement of autophagy in internalization and survival of HPVs was proved, the molecular mechanism is put forward. HPVs firstly bind to heparan-sulfonated proteoglycans of cell surface, following move to secondary receptors for internalization [54]. In keratinocytes, epidermal growth factor receptor (EGFR) and keratinocyte GFR (KGFR) are in response for sensing and transferring signals from HPV16 infection to activate the downstream pathways, of which the event is even prior to viral early protein expression [58-60]. As the infection process of HPVs primarily depends on autophagy inhibition, its infectivity might be directly enhanced by inhibiting the autophagy machinery. For example, 3-MA treatment could enhance infection levels, whereas tamoxifen abolished it [58].

Autophagy machinery also participates in bacteria and fungus infection on keratinocytes. Many pathogenic bacteria could produce the virulence factors of poreforming toxins (PFT) for infection. The membrane perforation is necessary for bacteria infection, and the accompanying efflux of ion would trigger sensors of starvation and energy depletion causing autophagic defense for recovery from sublethal attack. In HaCaT cells (an immortalized keratinocyte cell line), activation of AMPK, subsequent de-phosphorylation/ rephosphorylation kinetics of S6K and enhanced LC3 lipidation after toxin infection were observed. Formation of LC3-postive puncta and lipid modified form or replenishment of ATP were also inhibited by 3-MA in the alpha-toxin treated cells [61]. Fungus of Aspergillus and Penicillium produce patulin (PAT) for infection. PAT is a mycotoxin and shows carcinogenesis when associated with 12-tetradecanoyl phorbol myristate acetate (TPA) [62]. PAT treatment caused the inhibition of autophagosome degradation accompanied by the accumulation of p62 and consequent reactive oxygen species (ROS) generation in keratinocytes, ultimately disrupting apoptosis via Erk1/2 signaling [63]. Inhibited autophagy by PAT was also associated with reduced activities of lysosomal enzymes cathepsin B/D. The pro-survival signaling initiated by PAT-inhibited autophagy might contribute to the PATinduced skin carcinogenesis. Autophagy-related signaling in response to HPV, PFT and PAT infection are described in Figure 3.

\section{Role of autophagy in stress response}

As a housekeeping pathway, autophagy is vital for cells to survive various stresses such as nutrient deprivation, oxidative stress, hypoxia and pathogens [64]. Even though autophagy in response to UV is probably due to the deuterogenic oxidative stress, autophagy against UV radiation will be discussed individually.

$\mathrm{UV}$, an extremely common stressor from environment, leads to a series of morphological, ultrastructural and physiological alterations in human epidermis. Keratinocytes respond to the UV-induced damage either by repairing or tolerating it, ultimately undergoing programmed cell death. The electron microscopy result did not show apoptotic cells but the features of autophagy in the UV-treated cells suggesting that autophagy might be one of the cytoprotective mechanisms against the UV-induced apoptosis in keratinocytes [65]. Once some damaged cells escape apoptosis, autophagy may become vital to block canceration. The cells from SCC present higher autophagy level compared with normal keratinocytes, suggesting that the UV stress-induced autophagy might make sense in promoting carcinogenesis and tumor cell survival [66].

UV radiation is classified into UVA, UVB and UVC based on the wavelength, among which UVB is the main cause of skin damage. UVB could induce autophagy of human epidermal keratinocytes at earlier time points [66]. UVA could also induce autophagy in primary murine keratinocytes, but it seems that the UVA-induced autophagy plays an opposite effect compared to UVB. The ATG7-deleted keratinocytes irradiated by UVA showed the defective clearance of p62 and the elevation of nuclear factor-like 2 (Nrf2) target gene expression [67]. As the release of Nrf2 from cytosolic anchor usually cause tissue damage rather than protective responses, the UVA-induced autophagy is highly possible to promote cell death.

Oxidative stress enhanced autophagy is fatal to keratinocytes, which is demonstrated by the decreased death of senescent cells in the presence of 3-MA or antiATG5 siRNA treatment [68]. Even though organelles were damaged in the senescent cells, metabolic activity maintains somehow, as mitochondria increased in number. Notably, the apoptosis and autophagy induced by oxidative stress could be inhibited by nacetylcysteine (NAC), a ROS scavenger, indicating that ROS acts as the intermediate for apoptosis and autophagy. Meanwhile, glutathione (GSH) could mimic the protective effect of NAC [69].

Autophagy is also activated in response to stresses induced by hypoxia or ischemia followed by reoxygenation to remove non-functional or damaged cellular structures [70-72]. As we know, re-epithelialization which involves proliferation, differentiation and migration of the keratinocytes is critical for would healing process. Remifentanil, a drug widely used for general anesthesia induction and analgesia, could induce autophagy in keratinocytes and is proved to be beneficial to wound healing against hypoxiareoxygenation injury. Remifentanil could also recover the 3-MA-blocked autophagy which reveals the possible 
involvement of autophagy in would healing [73]. Lifting autophagic flux caused by hypoxia in the $\mathrm{HaCaT}$ cells also reduced the sensitivity of tumor necrosis factor-related apoptosis-inducing ligand (TRAIL), a toxicant against malignant cells, indicating that autophagy inhibitors could be employed combing with TRAIL in therapies against skin cancers [74].

\section{Role of autophagy in keratinocyte senescence}

Senescence is described as a kind of programmed cell death. The senescent cell with its own time schedule is cell-cycle arrested and displays morphological, metabolic and genetic changes differing from the dead or young one. Cell death now is classified into three types: apoptosis (type I), autophagy programmed death (type II) and necrosis (type III) [75, 76]. The senescent keratinocytes usually present extremely high autophagic activity characterized by an accumulation of a large number of autophagy vacuoles and a particular intracellular organization [77]. The autophagic programmed cell death is the primary mechanism of cell death for the senescent keratinocytes rather than apoptosis. Death of senescent cells was delayed when the initial phase of autophagy was blocked by 3-MA, without any change on apoptotic markers, meanwhile apoptotic inhibitors could not decrease the cell death in senescent cells [77]. However, apoptosis is likely to co-exist in low proportions with autophagy, of which the significance need to be clarified. Apoptosis may compromise autophagic cell death against oxidative stress, or increased autophagy might initiate apoptosis. Advancing age, telomere erosion and oxidative damage can lead to senescence [78]. Oxidative stress might be the key incentive factor. Intracellular accumulation of $\mathrm{H}_{2} \mathrm{O}_{2}$ could enhance autophagy to eliminate the oxidative-damaged mitochondria and nuclei, and initiate the senescence in keratinocytes and the program for final death [68].

In this sense, clarifying the role of autophagy in senescence would be of pathological and physiological significance. Then autophagy and senescence share the same marker of senescence-associated $\beta$-galactosidase (SA- $\beta-$ Gal) which is increased during aging [79, 80]. Together with the fact that oxidative stress could accelerate aging, we consider that there should be inevitable links among autophagy, senescence and aging.

Recently, it was reported that the Ras-Raf-Erkmediated autophagy and senescence is essential to prevent the skin tumorigenesis. Xie et al. [81] discovered that the activation of Kras initiated Erk signaling to induce a high level of ROS production, which down-regulated the activity of mTORC1 to enhance autophagy and senescence in the keratinocytes with normal protein expression of Sag. However, Sag deletion led to the accumulation of Erbin and Nrf2, two substrates of Sag, and subsequently caused the blockage of Ras-Raf signaling and clearance of ROS respectively. These events resulted in the relief of mTOR inactivation and blockage of autophagy and senescence. Accordingly, accelerated papillomagenesis has been observed in the skin of the $\mathrm{Sag}^{-/}$mice. Since escaping senescent-cell death might be a requisite step for tumor formation, application of possible autophagy inducers responsible for autophagic programmed cell death might be a therapy strategy through eliminating senescent cells.

\section{Autophagy determines keratinocyte fate, to survive or to die?}

In most instances, autophagy functions as a survival processor, though it may turn into the lethal machinery when inappropriate level of autophagy occurs. Cells digest themselves to resist adverse factors like metabolic stress induced by nutrient deprivation or energy deficiency. When autophagy is incapable to eliminate intracellular damage it may shift to the programmed cell death. For instance, autophagy will lead to a destructive outcome when parallel damage in lysosomal and mitochondrial membranes occurs [82]. Autophagic programmed cell death is characterized by accumulation of numerous autophagosomes and incontinent degradation of cytosolic components, whereas the nucleuses remain intact until late stage [83]. Even though beyond thirty ATG members have been reported to involve in the autophagy process, they act differently during "survival" autophagy and the "death" one. For example, levels of ATG5 and Beclin1 do not change during the starvation-induced autophagy but increase during the autophagic programmed cell death [84]. Conflicting with this finding, there comes an interesting phenomenon in the keratinocytes that Beclin1 indeed increases in comparison to the normal cells, even though the keratinocytes are of high basic autophagic activity which is enough for senescent cells to end up by death [68].

Both autophagy and apoptosis are in response for cell death as we mentioned before, and the two processes are intimately connected. In keratinocytes, activated autophagy plays an indispensable role of anti-apoptosis to promote cell survival against various stresses. For example, autophagy deficiency facilitates the activation of p38 pathway and subsequent apoptosis suggesting the anti-apoptotic action of autophagy [84]. A possible switch controlling autophagy and apoptosis in keratinocytes is recently described. In the canonical autophagy pathway, the increase of autophagy is accompanied by the dissociation of Beclin1 from myeloid cell leukemia-1 (Mcl-1) [85]. Mcl-1 can complex with Noxa, a proapoptotic effector belonging to Bcl-2 family, of which the activation trigger apoptosis after UVB irradiation in a p53-dependent or independent manner [86]. In the iASPP-depleted keratinocytes, the increase of Beclin1 and decrease of Mcl-1 and Noxa have been observed 
simultaneously [30]. Thus iASPP expression is proposed a switch controlling autophagy and apoptosis in this cell setting.

\section{THERAPEUTIC APPROACHES FOR SKIN DISEASES}

\section{Resveratrol}

Trace back to 1990 s, resveratrol was reported to have protective effect on skin against the UV-induced photodamage and photoaging [87]. Then further studies implicated the involvement of resveratrol in the regulation of autophagy partially due to its effects on redox balance [88]. Autophagy induced by resveratrol pre-treatment alone can be revealed by the reversible and irreversible suppression on the Akt-mTOR signaling and the increase of Beclin1 expression as well as the cell growth arrest which conforms to the canonical pro-survival autophagy in $\mathrm{HaCaT}$ cells [89]. However when associated with UVB of different dosages, the autophagy converted to a fatal one. Enhanced apoptosis and autophagy coexist in the keratinocytes pre-treated with resveratrol before UVB irradiation reducing the odds of non-tumorigenic cells to escape death. Because of the ability to interfere with multiple cellular pathways, resveratrol is promising for the prevention and therapy of hyperproliferative skin diseases like malignant cancers. For example, the premature senescence induced by resveratrol was associated with autophagic blockage via Rictor, a component of mTORC2 [90] in A431 epidermoid carcinoma cell line (malignant keratinocytes derived from SCC).

\section{Calcipotriol}

Calcipotriol, an analog of vitamin D, could induce autophagy in both normal and immortalized keratinocytes [91]. The vitamin D analogs have been used for the treatment of many skin diseases including psoriasis, recalcitrant warts, lamellar ichthyosis and epidermolytic hyperkeratosis [92-94]. Now recognizing the regulatory mechanism of calcipotriol on autophagy would be helpful to understand the pleiotropic effects of vitamin D analogs and vastly expand the application on the therapy for some skin disease, for example intracellular infection and hyperproliferation.

\section{$\alpha-S a n t a l o l$}

$\alpha$-Santalol, one of the primary components of sandalwood oil which commonly used as fragrances and incense, has been reported to have potential tumor prevention effect against UV exposure. $\alpha$-Santalol treatment could induce autophagy along with an increase of impaired plasma membrane integrity in the $\mathrm{HaCaT}$ cells, but not apoptosis [95]. Since the effect is more prominent in the proliferating cells than in the quiescent cells, cytotoxic treatment might be considered as an alternative way for the skin cancer therapy, which exerts less damage on normal cells via selective induction of autophagy.

\section{Apigenin}

Apigenin, a kind bioflavonoid, presents in variety of food sources and performs the antitumorigenic and chemopreventive actions [96]. The chemoprotective action of apigenin can be interpreted by the induction of autophagy via CaMK kinase- $\beta$ (CaMKK $\beta$ )-AMPKTSC2-mTOR and Akt-TSC2-mTOR signaling, but mainly through the activation of the former one in $\mathrm{HaCaT}$ cells [97]. Additionally, apigenin pretreatment could dramatically enhance the UVB-induced AMPK activation while UVB treatment alone just exerted moderate effect.

\section{Chromium and hexavalent chromium (Cr (VI))}

Chromium or hexavalent chromium (Cr (VI)) is ubiquitous in the environment and contributes to some skin diseases like chromium hypersensitivity and allergic contact dermatitis of which the occurrence is related to the ROS-mediated activated autophagy [98, 99]. In HaCaT cells, $\mathrm{Cr}$ (VI) treatment increased the LC3-II production, while combining with the pretreatment of NAC, a ROS scavenger, prohibited this effect. Moreover, the study on albino guinea pig model showed that NAC was curative for the chromium hypersensitivity [100]. The regulation details have not been described, but it can be speculated to involve in the Akt pathway.

\section{5-fluorouracil (5-FU)}

The antimetabolite 5-FU is commonly used in the therapy of colorectal cancer [101]. 5-FU inhibits thymidylate synthase enzyme to block DNA synthesis and also interferes with RNA processing. Similar to many other drugs, resistance is one of the major problems that retarded curative effects, of which the mechanisms involve alterations in related enzymes, p53, ataxia telangiectasia mutated kinase (ATM) or ataxia telangiectasia and Rad3related kinase (ATR). It was reported that activated p38 or resurgent autophagy mediates the resistance to 5 -FU in the HaCaT cells [102]. p38 is activated by 5 -FU through both ATM and ATR, and is dependent on its canonical adjacent up-stream signals, such as mitogen-activated protein kinase kinase (MAP2K), MAP kinase kinase 3 (MKK3) and MKK6. In the normal conditions, the 
genotoxic response triggered by 5-FU treatment promotes apoptosis and blocks autophagy. Nevertheless, when p38 is blocked, the increase of autophagy with the decrease of apoptosis rendered the resistant phenotype. Therefore, $5-\mathrm{FU}$ is a crucial regulator controlling the balance between apoptosis and autophagy to determine cell fate. Levofolene (LF), often used in combination with 5-FU, showed an antagonistic effect on autophagy to modulate apoptosis elicited by 5-FU treatment [103]. Therefore, the inhibition of 5-FU induced autophagy contributed to the enhancement of toxicity on keratinocytes treated with 5-FU in combined with LF treatment, which can produce a severe dermal side effect, for example handfoot syndrome.

\section{Efavirenz (EFV)}

EFV is commonly used for the antiretroviral therapy, but usually with the adverse drug reaction of cutaneous and mucosal lesions [104]. The keratinocytes exposed to EFV exhibited a decrease of viability and premature terminal differentiation, leading to the impaired epithelial regeneration and subsequent atrophy [105]. Assessment of the related intracellular signaling showed the depressed mTOR signaling and activated Erk1/2 signaling together with rapid p53 degradation, indicating that the inhibitory effect of EFV on epithelial regeneration is related to the autophagy activation.

\section{Oligosaccharides}

Some nature oligosaccharides such as trehalose, sucrose and raffinose were reported to be cargos of autophagosomes. Noticeably, trehalose exhibited a therapeutic effect through an mTOR-independent induction of autophagy in preventing neural tube defects, retarding the progression of amyotrophic lateral sclerosis, alleviating the dopaminergic and tau pathology, resisting the cellular prion infection and promoting the clearance of mutant huntingtin and alpha-synuclein [106-110]. Our recent work revealed that trehalose, sucrose, and raffinose can enhance autophagy in human keratinocytes via the mTOR-independent way, and trehalose treatment decreases cell death and prohibits the abnormal cell proliferation caused by UV stimulation (data have not been published).

\section{CONCLUSIONS}

As a highly conserved intracellular mechanism for homeostasis maintenance, autophagy is proved to participate in series of physiological activities in keratinocytes, including apoptosis, differentiation, inflammation and melanin metabolism. Autophagy is even closely related to senescence, aging, skin color formation, and also displays the ability of anti-stress and anti-infection. Most notably, it distinctly functions as the switch for controlling the cell death, as some times autophagy presents a cytoprotective talent, but it shifts to a fatal mechanism in some conditions. Thus, application of autophagy regulators in keratinocytes implied a promising strategy for the therapy of abundant skin diseases.

However, the cells with defects in autophagy also showed persistent DNA damage and increased chromosome instability, while excessively activated autophagy might also leads to adverse results. Therefore, much works are needed on optimizing designed strategies to inhibit or enhance autophagy for clinical efficacy, since autophagy routinely plays the role of a double-edged sword in various conditions, which can be illustrated by the studies on the performances of autophagy in cancer. On one hand, autophagy acts as a tumor suppressor to clear damaged organelles and accumulated growth factors and maintain chromosomal stability. In this case, Beclin1 and ATG5 have been identified as the "guardian" for the cellular genome [111]. On the other hand, the cytoprotective effect of autophagy on cancer cells through damage elimination and energy supply would be helpful to resist anticancer therapy [112]. Now, autophagy inhibitors and activators were both applied combining with anticancer agents to reinforce the efficiency [113]. Indeed, autophagy not only eliminates the damage caused by anticancer treatment, but also provides the energy for cell division. For example, autophagy is activated in the cancer cells which are in circumstance of hypoxia, a common condition in solid tumor [114]. Under this nonphysiological event, autophagy is useful to sustain the cancer cells' proliferation through degrading proteins, DNA and organelles and maintaining their energy provision. Thus, the issue of how to manipulate autophagy to fight against tumorigenesis remains extremely intriguing.

Series of evidences summarized in this review demonstrated the extremely complicated role of autophagy in cutaneous physiology and pathology. However, there remains questions impeding further clarification of the role of autophagy in keratinocytes: (i) The basal autophagy level is distinct in the different keratinocyte settings like primary keratinocytes and $\mathrm{HaCaT}$ cells, as well as epidermal cancer cell line (e.g. A431), possibly due to different culture conditions (culture medium with or without serum) or origins of human races. (ii) Studies in vivo were extremely limited, particularly on the performance of autophagy in common skin disorders associated with keratinocytes. (iii) Unique autophagy machinery probably exists in human keratinocytes.

About forty ATG genes have been identified in the mammalian cells and/or yeast without complete confirmation of their functions. The ATG proteins in keratinocytes remain to be clarified for their expression regulation, protein-protein interaction, roles in autophagic 
machinery and extended functions. Recently, Kemp et al. reported that ULK1 is deregulated due to the UVinduced DNA damage in keratinocytes, which caused a stimulator of interferon genes (STING)-dependent interferon regulatory factor 3 (IRF3) activation, suggesting the function of ULK1 in immune response [115]. Besides the autophagy regulation, ATG proteins perform other biological functions such as inflammation, immunity and efferocytosis. Therefore, we consider that clarification of distinct performances of the ATG proteins in keratinocytes is essential to demonstrate the biological roles of autophagy in skin disorders.

At last and notably, besides series of signaling effectors we mentioned above, microRNAs (miRNAs), a group of RNA nucleotides with the function of modulating mRNA translation/degradation, emerge as promising targets for cancer therapy, and numerous evidences have indicated the role of miRNAs in cell biology [118, 119]. Recently numbers of miRNAs were found exposing their tight links to autophagy pathway. For instance, autophagy contributes to miRNA activity and homeostasis by regulating the degradation of cytosolic miRNA-containing complexes or modulating miRNA-mediated gene silencing $[120,121]$. Accordingly, miRNAs were reported to involve in autophagy regulation by directly targeting proteins associated with autophagy like Beclin1, Atg4C, ATG5, FAK family-interacting protein of $200 \mathrm{kDa}$ (FIP200), ATG7 and even mTOR [122-124]. However, there is very a few reports yet on interaction performance of miRNAs and autophagy in keratinocytes, most studies devoted to understanding miRNA performances in keratinocyte proliferation, migration, differentiation and senescence and without description of autophagy involvement [125127]. It's exciting that if combing with available results we have described in this review above, these limited results would be inspiring. For instance, Rivetti et al. reported the contribution of miR-138, $-181 \mathrm{a},-181 \mathrm{~b}$ and $-130 \mathrm{~b}$ to induction of senescence in keratinocytes by modulating levels of p63 (belong to p53 family) and silent matingtype information regulation 2 homologue 1 (Sirt1) [126]. Considering the interplay between p53 or Sirt1 and autophagy, and the relationship between autophagy and senescence in keratinocytes have already been revealed, we could speculate that autophagy could act the intermediation role between miRNAs and senescence $[27,30,105]$. Conclusively, considering the widespread significance of miRNAs in cell biology regulation and increasing evidences revealing the tight involvement in autophagy, clarifying relationship between miRNAs and autophagy in keratinocytes is needed more investigation in depth.

Drugs targeting autophagy modulation have been followed with the interests in treatment for cancer, infection and neurodegenerative diseases. For instance, autophagy blocker chloroquine and its analogue of hydroxychloroquine have been exploited in series clinical trials to potentiate anticancer therapy $[128$, 129]. Importantly, chloroquine and hydroxychloroquine are widely used in the therapy for many kinds of skin disorders such as photosensitivity dermatosis, lupus erythematosus and lichen planus. However, it remains to confirm whether autophagy affected by treatment of them is related to the therapeutic effect against these diseases. In a word, the agents targeting autophagy modulation are extremely potential for widely application in dermatology in the individual or combination manner.

\section{ACKNOWLEDGMENTS}

This work was supported by grants from National Natural Science Foundation of China (No. 81171513, 81371755), PhD Programs Foundation of Ministry of Education of China (No. 20111106110041, 20131106120046), Funded by Jiangsu Provincial Special Program of Medical Science( BL2012003) and Jiangsu Province Natural Science Foundation (No. BK20131064) to Heng $\mathrm{Gu}$. Xu Chen is supported by PUMC Youth Fund and Fundamental Research Funds for the Central Universities (3332014008).

\section{CONFLICTS OF INTEREST}

There is no conflict of interest.

\section{REFERENCES}

1. White E, Karp C, Strohecker AM, Guo Y and Mathew R. Role of autophagy in suppression of inflammation and cancer. Current opinion in cell biology. 2010; 22:212-217.

2. Kondo Y, Kanzawa T, Sawaya R and Kondo S. The role of autophagy in cancer development and response to therapy. Nature reviews Cancer. 2005; 5:726-734.

3. Mizushima N and Komatsu M. Autophagy: renovation of cells and tissues. Cell. 2011; 147:728-741.

4. Cuervo AM. Autophagy: in sickness and in health. Trends in cell biology. 2004; 14:70-77.

5. Levine B and Kroemer G. Autophagy in the pathogenesis of disease. Cell. 2008; 132:27-42.

6. Mochida K, Oikawa Y, Kimura Y, Kirisako H, Hirano H, Ohsumi Y and Nakatogawa H. Receptor-mediated selective autophagy degrades the endoplasmic reticulum and the nucleus. Nature. 2015; 522:359-362.

7. Xie Z and Klionsky DJ. Autophagosome formation: core machinery and adaptations. Nature cell biology. 2007; 9:1102-1109.

8. Mizushima N. Autophagy: process and function. Genes \& development. 2007; 21:2861-2873.

9. Tripathi DN, Chowdhury R, Trudel LJ, Tee AR, Slack RS, Walker CL and Wogan GN. Reactive nitrogen species regulate autophagy through ATM-AMPK-TSC2-mediated 
suppression of mTORC1. Proceedings of the National Academy of Sciences of the United States of America. 2013; 110:E2950-2957.

10. Gwinn DM, Shackelford DB, Egan DF, Mihaylova MM, Mery A, Vasquez DS, Turk BE and Shaw RJ. AMPK phosphorylation of raptor mediates a metabolic checkpoint. Molecular cell. 2008; 30:214-226.

11. Kim J, Kundu M, Viollet B and Guan KL. AMPK and mTOR regulate autophagy through direct phosphorylation of Ulk1. Nature cell biology. 2011; 13:132-141.

12. Li X, Lu Y, Lu H, Luo J, Hong Y and Fan Z. AMPKmediated energy homeostasis and associated metabolic effects on cancer cell response and resistance to cetuximab. Oncotarget. 2015; 6:11507-11518. doi: 10.18632/ oncotarget.3432.

13. Wong AK, Howie J, Petrie JR and Lang CC. AMP-activated protein kinase pathway: a potential therapeutic target in cardiometabolic disease. Clinical science. 2009; 116:607620.

14. Ha J, Guan KL and Kim J. AMPK and autophagy in glucose/glycogen metabolism. Molecular aspects of medicine. 2015; 46:46-62.

15. Hardie DG. AMPK and autophagy get connected. The EMBO journal. 2011; 30:634-635.

16. Mizushima N, Noda T, Yoshimori T, Tanaka Y, Ishii T, George MD, Klionsky DJ, Ohsumi M and Ohsumi Y. A protein conjugation system essential for autophagy. Nature. 1998; 395:395-398.

17. Fujita $\mathrm{N}$, Itoh $\mathrm{T}$, Omori $\mathrm{H}$, Fukuda $\mathrm{M}$, Noda $\mathrm{T}$ and Yoshimori T. The Atg16L complex specifies the site of LC3 lipidation for membrane biogenesis in autophagy. Molecular biology of the cell. 2008; 19:2092-2100.

18. Wu J, Dang Y, Su W, Liu C, Ma H, Shan Y, Pei Y, Wan $\mathrm{B}$, Guo J and Yu L. Molecular cloning and characterization of rat LC3A and LC3B-two novel markers of autophagosome. Biochemical and biophysical research communications. 2006; 339:437-442.

19. Carroll B, Mohd-Naim N, Maximiano F, Frasa MA, McCormack J, Finelli M, Thoresen SB, Perdios L, Daigaku R, Francis RE, Futter C, Dikic I and Braga VM. The TBC/ RabGAP Armus coordinates Rac1 and Rab7 functions during autophagy. Developmental cell. 2013; 25:15-28.

20. Frasa MA, Maximiano FC, Smolarczyk K, Francis RE, Betson ME, Lozano E, Goldenring J, Seabra MC, Rak A, Ahmadian MR and Braga VM. Armus is a Rac1 effector that inactivates Rab7 and regulates E-cadherin degradation. CB. 2010; 20:198-208.

21. Mack NA, Whalley HJ, Castillo-Lluva S and Malliri A. The diverse roles of Rac signaling in tumorigenesis. Cell cycle. 2011; 10:1571-1581.

22. Yao H, Shi W, Wu J, Xu C, Wang J, Shao Y, Wu X and Zhang Z. Endothelial Rac1 is essential for hematogenous metastasis to the lung. Oncotarget. 2015; 6:17501-17513. doi: 10.18632/oncotarget.3766.

23. Weidberg H, Shvets E and Elazar Z. Biogenesis and cargo selectivity of autophagosomes. Annual review of biochemistry. 2011; 80:125-156.

24. Pankiv S, Clausen TH, Lamark T, Brech A, Bruun JA, Outzen H, Overvatn A, Bjorkoy $\mathrm{G}$ and Johansen $\mathrm{T}$. p62/SQSTM1 binds directly to Atg8/LC3 to facilitate degradation of ubiquitinated protein aggregates by autophagy. The Journal of biological chemistry. 2007; 282:24131-24145.

25. Isakson P, Holland $P$ and Simonsen A. The role of ALFY in selective autophagy. Cell death and differentiation. 2013; 20:12-20.

26. He R, Peng J, Yuan P, Xu F and Wei W. Divergent roles of BECN1 in LC3 lipidation and autophagosomal function. Autophagy. 2015; 11:740-747.

27. Aymard E, Barruche V, Naves T, Bordes S, Closs B, Verdier $M$ and Ratinaud MH. Autophagy in human keratinocytes: an early step of the differentiation? Experimental dermatology. 2011; 20:263-268.

28. Marquez RT and Xu L. Bcl-2:Beclin 1 complex: multiple, mechanisms regulating autophagy/apoptosis toggle switch. American journal of cancer research. 2012; 2:214-221.

29. Djavaheri-Mergny M, Maiuri MC and Kroemer G. Cross talk between apoptosis and autophagy by caspase-mediated cleavage of Beclin 1. Oncogene. 2010; 29:1717-1719.

30. Chikh A, Sanza P, Raimondi C, Akinduro O, Warnes G, Chiorino G, Byrne C, Harwood CA and Bergamaschi D. iASPP is a novel autophagy inhibitor in keratinocytes. Journal of cell science. 2014; 127:3079-3093.

31. Rossiter H, Konig U, Barresi C, Buchberger M, Ghannadan M, Zhang CF, Mlitz V, Gmeiner R, Sukseree S, Fodinger D, Eckhart L and Tschachler E. Epidermal keratinocytes form a functional skin barrier in the absence of Atg7 dependent autophagy. Journal of dermatological science. 2013; 71:6775.

32. Douroudis K, Kingo K, Traks T, Reimann E, Raud K, Ratsep R, Mossner R, Silm H, Vasar E and Koks S. Polymorphisms in the ATG16L1 gene are associated with psoriasis vulgaris. Acta dermato-venereologica. 2012; 92:85-87.

33. Buckingham EM, Carpenter JE, Jackson W, Zerboni L, Arvin AM and Grose C. Autophagic flux without a block differentiates varicella-zoster virus infection from herpes simplex virus infection. Proceedings of the National Academy of Sciences of the United States of America. 2015; 112:256-261.

34. Choi SR, Chung BY, Kim SW, Kim CD, Yun WJ, Lee MW, Choi JH and Chang SE. Activation of autophagic pathways is related to growth inhibition and senescence in cutaneous squamous cell carcinoma. Experimental dermatology. 2014; 23:718-724.

35. Feldmeyer L, Hofbauer GF, Boni T, French LE and Hafner 
J. Mammalian target of rapamycin (mTOR) inhibitors slow skin carcinogenesis, but impair wound healing. The British journal of dermatology. 2012; 166:422-424.

36. Chatterjea SM, Resing KA, Old W, Nirunsuksiri W and Fleckman P. Optimization of filaggrin expression and processing in cultured rat keratinocytes. Journal of dermatological science. 2011; 61:51-59.

37. Haruna K, Suga Y, Muramatsu S, Taneda K, Mizuno Y, Ikeda S, Ueno T, Kominami E, Tanida I, Tanida I and Hanada K. Differentiation-specific expression and localization of an autophagosomal marker protein (LC3) in human epidermal keratinocytes. Journal of dermatological science. 2008; 52:213-215.

38. Yoshihara N, Ueno T, Takagi A, Oliva Trejo JA, Haruna K, Suga Y, Komatsu M, Tanaka K and Ikeda S. The significant role of autophagy in the granular layer in normal skin differentiation and hair growth. Archives of dermatological research. 2015; 307:159-169.

39. Belleudi F, Purpura V, Caputo S and Torrisi MR. FGF7/ KGF regulates autophagy in keratinocytes: A novel dual role in the induction of both assembly and turnover of autophagosomes. Autophagy. 2014; 10:803-821.

40. Soma-Nagae T, Nada S, Kitagawa M, Takahashi Y, Mori $\mathrm{S}$, Oneyama C and Okada M. The lysosomal signaling anchor p18/LAMTOR1 controls epidermal development by regulating lysosome-mediated catabolic processes. Journal of cell science. 2013; 126:3575-3584.

41. Takahashi Y, Nada S, Mori S, Soma-Nagae T, Oneyama $\mathrm{C}$ and Okada M. The late endosome/lysosome-anchored p18-mTORC1 pathway controls terminal maturation of lysosomes. Biochemical and biophysical research communications. 2012; 417:1151-1157.

42. Moriyama M, Moriyama H, Uda J, Matsuyama A, Osawa $\mathrm{M}$ and Hayakawa T. BNIP3 plays crucial roles in the differentiation and maintenance of epidermal keratinocytes. The Journal of investigative dermatology. 2014; 134:16271635.

43. Murase D, Hachiya A, Takano K, Hicks R, Visscher MO, Kitahara T, Hase T, Takema Y and Yoshimori T. Autophagy has a significant role in determining skin color by regulating melanosome degradation in keratinocytes. The Journal of investigative dermatology. 2013; 133:24162424.

44. Ebanks JP, Koshoffer A, Wickett RR, Schwemberger S, Babcock G, Hakozaki T and Boissy RE. Epidermal keratinocytes from light vs. dark skin exhibit differential degradation of melanosomes. The Journal of investigative dermatology. 2011; 131:1226-1233.

45. Belleudi F, Purpura V, Scrofani C, Persechino F, Leone $\mathrm{L}$ and Torrisi MR. Expression and signaling of the tyrosine kinase FGFR2b/KGFR regulates phagocytosis and melanosome uptake in human keratinocytes. FASEB journal. 2011; 25:170-181.
46. Cardinali G, Bolasco G, Aspite N, Lucania G, Lotti LV, Torrisi MR and Picardo M. Melanosome transfer promoted by keratinocyte growth factor in light and dark skin-derived keratinocytes. The Journal of investigative dermatology. 2008; 128:558-567.

47. Belleudi F, Leone L, Aimati L, Stirparo MG, Cardinali G, Marchese C, Frati L, Picardo M and Torrisi MR. Endocytic pathways and biological effects induced by UVB-dependent or ligand-dependent activation of the keratinocyte growth factor receptor. FASEB journal. 2006; 20:395-397.

48. Lee HM, Shin DM, Yuk JM, Shi G, Choi DK, Lee SH, Huang SM, Kim JM, Kim CD, Lee JH and Jo EK. Autophagy negatively regulates keratinocyte inflammatory responses via scaffolding protein p62/SQSTM1. Journal of immunology. 2011; 186:1248-1258.

49. Sun W, Zheng Y, Lu Z, Cui Y, Tian Q, Xiao S, Liu F and Liu J. Overexpression of S100A7 protects LPS-induced mitochondrial dysfunction and stimulates IL-6 and IL-8 in HaCaT cells. PloS one. 2014; 9:e92927.

50. Kollisch G, Kalali BN, Voelcker V, Wallich R, Behrendt H, Ring J, Bauer S, Jakob T, Mempel M and Ollert M. Various members of the Toll-like receptor family contribute to the innate immune response of human epidermal keratinocytes. Immunology. 2005; 114:531-541.

51. Miller LS. Toll-like receptors in skin. Advances in dermatology. 2008; 24:71-87.

52. Eckert RL, Broome AM, Ruse M, Robinson N, Ryan D and Lee K. S100 proteins in the epidermis. The Journal of investigative dermatology. 2004; 123:23-33.

53. Soong G, Paulino F, Wachtel S, Parker D, Wickersham M, Zhang D, Brown A, Lauren C, Dowd M, West E, Horst B, Planet $\mathrm{P}$ and Prince A. Methicillin-resistant Staphylococcus aureus adaptation to human keratinocytes. mBio. 2015; 6 .

54. Surviladze Z, Dziduszko A and Ozbun MA. Essential roles for soluble virion-associated heparan sulfonated proteoglycans and growth factors in human papillomavirus infections. PLoS pathogens. 2012; 8:e1002519.

55. Schelhaas M, Shah B, Holzer M, Blattmann P, Kuhling L, Day PM, Schiller JT and Helenius A. Entry of human papillomavirus type 16 by actin-dependent, clathrin- and lipid raft-independent endocytosis. PLoS pathogens. 2012; 8:e1002657.

56. Zhou $X$ and Munger K. Expression of the human papillomavirus type 16 E7 oncoprotein induces an autophagy-related process and sensitizes normal human keratinocytes to cell death in response to growth factor deprivation. Virology. 2009; 385:192-197.

57. Hanning JE, Saini HK, Murray MJ, Caffarel MM, van Dongen S, Ward D, Barker EM, Scarpini CG, Groves IJ, Stanley MA, Enright AJ, Pett MR and Coleman N. Depletion of HPV16 early genes induces autophagy and senescence in a cervical carcinogenesis model, regardless of viral physical state. The Journal of pathology. 2013; 
231:354-366

58. Surviladze Z, Sterk RT, DeHaro SA and Ozbun MA. Cellular entry of human papillomavirus type 16 involves activation of the phosphatidylinositol 3-kinase/Akt/mTOR pathway and inhibition of autophagy. Journal of virology. 2013; 87:2508-2517.

59. Belleudi F, Nanni M, Raffa S and Torrisi MR. HPV16 E5 deregulates the autophagic process in human keratinocytes. Oncotarget. 2015; 6:9370-9386. doi: 10.18632/ oncotarget.3326.

60. Purpura V, Belleudi F, Caputo S and Torrisi MR. HPV16 E5 and KGFR/FGFR2b interplay in differentiating epithelial cells. Oncotarget. 2013; 4:192-205. doi: 10.18632/ oncotarget.803.

61. Kloft N, Neukirch C, Bobkiewicz W, Veerachato G, Busch T, von Hoven G, Boller K and Husmann M. Pro-autophagic signal induction by bacterial pore-forming toxins. Medical microbiology and immunology. 2010; 199:299-309.

62. Saxena N, Ansari KM, Kumar R, Chaudhari BP, Dwivedi $\mathrm{PD}$ and Das M. Role of mitogen activated protein kinases in skin tumorigenicity of patulin. Toxicology and applied pharmacology. 2011; 257:264-271.

63. Guo X, Dong Y, Yin S, Zhao C, Huo Y, Fan L and Hu $\mathrm{H}$. Patulin induces pro-survival functions via autophagy inhibition and p62 accumulation. Cell death \& disease. 2013; 4:e822.

64. Kroemer G, Marino G and Levine B. Autophagy and the integrated stress response. Molecular cell. 2010; 40:280293.

65. Misovic M, Milenkovic D, Martinovic T, Ciric D, Bumbasirevic V and Kravic-Stevovic T. Short-term exposure to UV-A, UV-B, and UV-C irradiation induces alteration in cytoskeleton and autophagy in human keratinocytes. Ultrastructural pathology. 2013; 37:241-248.

66. Qiang L, Wu C, Ming M, Viollet B and He YY. Autophagy controls p38 activation to promote cell survival under genotoxic stress. The Journal of biological chemistry. 2013; 288:1603-1611.

67. Zhao Y, Zhang CF, Rossiter H, Eckhart L, Konig U, Karner S, Mildner M, Bochkov VN, Tschachler E and Gruber F. Autophagy is induced by UVA and promotes removal of oxidized phospholipids and protein aggregates in epidermal keratinocytes. The Journal of investigative dermatology. 2013; 133:1629-1637.

68. Deruy E, Gosselin K, Vercamer C, Martien S, Bouali F, Slomianny C, Bertout J, Bernard D, Pourtier A and Abbadie C. MnSOD upregulation induces autophagic programmed cell death in senescent keratinocytes. PloS one. 2010; 5:e12712.

69. Yu Y, Fan SM, Song JK, Tashiro S, Onodera S and Ikejima T. Hydroxyl radical (.OH) played a pivotal role in oridonininduced apoptosis and autophagy in human epidermoid carcinoma A431 cells. Biological \& pharmaceutical bulletin. 2012; 35:2148-2159.
70. Li X, Zeng Z, Li Q, Xu Q, Xie J, Hao H, Luo G, Liao W, Bin J, Huang X and Liao Y. Inhibition of microRNA-497 ameliorates anoxia/reoxygenation injury in cardiomyocytes by suppressing cell apoptosis and enhancing autophagy. Oncotarget. 2015; 6:18829-18844. doi: 10.18632/ oncotarget. 4774 .

71. Wang B, Zhong S, Zheng F, Zhang Y, Gao F, Chen Y, Lu $\mathrm{B}, \mathrm{Xu} \mathrm{H}$ and Shi G. N-n-butyl haloperidol iodide protects cardiomyocytes against hypoxia/reoxygenation injury by inhibiting autophagy. Oncotarget. 2015; 6:24709-24721. doi: 10.18632/oncotarget.5077.

72. Pursiheimo JP, Rantanen K, Heikkinen PT, Johansen T and Jaakkola PM. Hypoxia-activated autophagy accelerates degradation of SQSTM1/p62. Oncogene. 2009; 28:334-344.

73. Kwon JY, Park BS, Kim YH, Kim YD, Kim CH, Yoon JY and Yoon JU. Remifentanil protects human keratinocytes against hypoxia-reoxygenation injury through activation of autophagy. PloS one. 2015; 10:e116982.

74. Kim SW and Park SY. Hypoxiamediated activation of autophagic flux inhibits apoptosis of keratinocytes via blocking tumor necrosis factorrelated apoptosisinducing ligand. Molecular medicine reports. 2015.

75. Mancias JD and Kimmelman AC. Targeting autophagy addiction in cancer. Oncotarget. 2011; 2:1302-1306. doi: 10.18632/oncotarget.384.

76. Lockshin RA and Zakeri Z. Apoptosis, autophagy, and more. The international journal of biochemistry \& cell biology. 2004; 36:2405-2419.

77. Gosselin K, Deruy E, Martien S, Vercamer C, Bouali F, Dujardin T, Slomianny C, Houel-Renault L, Chelli F, De Launoit $Y$ and Abbadie C. Senescent keratinocytes die by autophagic programmed cell death. The American journal of pathology. 2009; 174:423-435.

78. Cristofalo VJ, Lorenzini A, Allen RG, Torres C and Tresini M. Replicative senescence: a critical review. Mechanisms of ageing and development. 2004; 125:827-848.

79. Dimri GP, Lee X, Basile G, Acosta M, Scott G, Roskelley C, Medrano EE, Linskens M, Rubelj I, Pereira-Smith O and et al. A biomarker that identifies senescent human cells in culture and in aging skin in vivo. Proceedings of the National Academy of Sciences of the United States of America. 1995; 92:9363-9367.

80. Keyes WM, Wu Y, Vogel H, Guo X, Lowe SW and Mills AA. p63 deficiency activates a program of cellular senescence and leads to accelerated aging. Genes \& development. 2005; 19:1986-1999.

81. Xie CM, Wei D, Zhao L, Marchetto S, Mei L, Borg JP and Sun Y. Erbin is a novel substrate of the Sag-betaTrCP E3 ligase that regulates KrasG12D-induced skin tumorigenesis. The Journal of cell biology. 2015; 209:721-737.

82. Martins WK, Costa ET, Cruz MC, Stolf BS, Miotto R, Cordeiro RM and Baptista MS. Parallel damage in mitochondrial and lysosomal compartments promotes efficient cell death with autophagy: The case of the 
pentacyclic triterpenoids. Scientific reports. 2015; 5:12425.

83. Klionsky DJ. Autophagy: from phenomenology to molecular understanding in less than a decade. Nature reviews Molecular cell biology. 2007; 8:931-937.

84. Shimizu S, Kanaseki T, Mizushima N, Mizuta T, ArakawaKobayashi S, Thompson CB and Tsujimoto Y. Role of Bcl2 family proteins in a non-apoptotic programmed cell death dependent on autophagy genes. Nature cell biology. 2004; 6:1221-1228.

85. Maiuri MC, Criollo A, Tasdemir E, Vicencio JM, Tajeddine N, Hickman JA, Geneste O and Kroemer G. $\mathrm{BH} 3$-only proteins and $\mathrm{BH} 3$ mimetics induce autophagy by competitively disrupting the interaction between Beclin 1 and Bcl-2/Bcl-X(L). Autophagy. 2007; 3:374-376.

86. Naik E, Michalak EM, Villunger A, Adams JM and Strasser A. Ultraviolet radiation triggers apoptosis of fibroblasts and skin keratinocytes mainly via the BH3-only protein Noxa. The Journal of cell biology. 2007; 176:415-424.

87. Jang M, Cai L, Udeani GO, Slowing KV, Thomas CF, Beecher $\mathrm{CW}$, Fong $\mathrm{HH}$, Farnsworth NR, Kinghorn AD, Mehta RG, Moon RC and Pezzuto JM. Cancer chemopreventive activity of resveratrol, a natural product derived from grapes. Science. 1997; 275:218-220.

88. Pervaiz S and Holme AL. Resveratrol: its biologic targets and functional activity. Antioxidants \& redox signaling. 2009; 11:2851-2897.

89. Vitale N, Kisslinger A, Paladino S, Procaccini C, Matarese G, Pierantoni GM, Mancini FP and Tramontano D. Resveratrol couples apoptosis with autophagy in UVBirradiated HaCaT cells. PloS one. 2013; 8:e80728.

90. Back JH, Zhu Y, Calabro A, Queenan C, Kim AS, Arbesman $\mathrm{J}$ and Kim AL. Resveratrol-mediated downregulation of Rictor attenuates autophagic process and suppresses UV-induced skin carcinogenesis. Photochemistry and photobiology. 2012; 88:1165-1172.

91. Wang RC and Levine B. Calcipotriol induces autophagy in HeLa cells and keratinocytes. The Journal of investigative dermatology. 2011; 131:990-993.

92. Egawa $\mathrm{K}$ and Ono T. Topical vitamin D3 derivatives for recalcitrant warts in three immunocompromised patients. The British journal of dermatology. 2004; 150:374-376.

93. Umekoji A, Fukai K and Ishii M. A case of mosaic-type bullous congenital ichthyosiform erythroderma successfully treated with topical maxacalcitol, a vitamin D3 analogue. Clinical and experimental dermatology. 2008; 33:501-502.

94. Ito K, Koga M, Shibayama Y, Tatematsu S, Nakayama $\mathrm{J}$ and Imafuku S. Proactive treatment with calcipotriol reduces recurrence of plaque psoriasis. The Journal of dermatology. 2015.

95. Dickinson SE, Olson ER, Levenson C, Janda J, Rusche JJ, Alberts DS and Bowden GT. A novel chemopreventive mechanism for a traditional medicine: East Indian sandalwood oil induces autophagy and cell death in proliferating keratinocytes. Archives of biochemistry and biophysics. 2014; 558:143-152.

96. Abu-Yousif AO, Smith KA, Getsios S, Green KJ, Van Dross RT and Pelling JC. Enhancement of UVB-induced apoptosis by apigenin in human keratinocytes and organotypic keratinocyte cultures. Cancer research. 2008; 68:3057-3065.

97. Tong X, Smith KA and Pelling JC. Apigenin, a chemopreventive bioflavonoid, induces AMP-activated protein kinase activation in human keratinocytes. Molecular carcinogenesis. 2012; 51:268-279.

98. Lee YH, Su SB, Huang CC, Sheu HM, Tsai JC, Lin CH, Wang YJ and Wang BJ. N-acetylcysteine attenuates hexavalent chromium-induced hypersensitivity through inhibition of cell death, ROS-related signaling and cytokine expression. PloS one. 2014; 9:e108317.

99. Bickers DR and Athar M. Oxidative stress in the pathogenesis of skin disease. The Journal of investigative dermatology. 2006; 126:2565-2575.

100. Wang BJ, Guo YL, Chang HY, Sheu HM, Pan MH, Lee YH and Wang YJ. N-acetylcysteine inhibits chromium hypersensitivity in coadjuvant chromium-sensitized albino guinea pigs by suppressing the effects of reactive oxygen species. Experimental dermatology. 2010; 19:e191-200.

101. Srinivas US, Dyczkowski J, Beissbarth T, Gaedcke $\mathrm{J}$, Mansour WY, Borgmann $\mathrm{K}$ and Dobbelstein $\mathrm{M}$. 5-Fluorouracil sensitizes colorectal tumor cells towards double stranded DNA breaks by interfering with homologous recombination repair. Oncotarget. 2015; 6:12574-12586. doi: 10.18632/oncotarget.3728.

102. de la Cruz-Morcillo MA, Valero ML, Callejas-Valera JL, Arias-Gonzalez L, Melgar-Rojas P, Galan-Moya EM, Garcia-Gil E, Garcia-Cano J and Sanchez-Prieto R. P38MAPK is a major determinant of the balance between apoptosis and autophagy triggered by 5-fluorouracil: implication in resistance. Oncogene. 2012; 31:1073-1085.

103. Lamberti M, Porto S, Zappavigna S, Stiuso P, Tirino V, Desiderio V, Mele L and Caraglia M. Levofolene modulates apoptosis induced by 5-fluorouracil through autophagy inhibition: clinical and occupational implications. International journal of oncology. 2015; 46:1893-1900.

104. Borras-Blasco J, Navarro-Ruiz A, Borras C and Castera E. Adverse cutaneous reactions associated with the newest antiretroviral drugs in patients with human immunodeficiency virus infection. The Journal of antimicrobial chemotherapy. 2008; 62:879-888.

105. Dong Q, Oh JE, Yi JK, Kim RH, Shin KH, Mitsuyasu R, Park NH and Kang MK. Efavirenz induces autophagy and aberrant differentiation in normal human keratinocytes. International journal of molecular medicine. 2013; 31:13051312.

106. Xu C, Li X, Wang F, Weng $\mathrm{H}$ and Yang P. Trehalose 
prevents neural tube defects by correcting maternal diabetes-suppressed autophagy and neurogenesis. American journal of physiology Endocrinology and metabolism. 2013; 305:E667-678.

107. Castillo K, Nassif M, Valenzuela V, Rojas F, Matus S, Mercado G, Court FA, van Zundert B and Hetz C. Trehalose delays the progression of amyotrophic lateral sclerosis by enhancing autophagy in motoneurons. Autophagy. 2013; 9:1308-1320.

108. Rodriguez-Navarro JA, Rodriguez L, Casarejos MJ, Solano RM, Gomez A, Perucho J, Cuervo AM, Garcia de Yebenes $\mathrm{J}$ and Mena MA. Trehalose ameliorates dopaminergic and tau pathology in parkin deleted/tau overexpressing mice through autophagy activation. Neurobiology of disease. 2010; 39:423-438.

109. Aguib Y, Heiseke A, Gilch S, Riemer C, Baier M, Schatzl HM and Ertmer A. Autophagy induction by trehalose counteracts cellular prion infection. Autophagy. 2009; 5:361-369.

110. Sarkar S, Davies JE, Huang Z, Tunnacliffe A and Rubinsztein DC. Trehalose, a novel mTOR-independent autophagy enhancer, accelerates the clearance of mutant huntingtin and alpha-synuclein. The Journal of biological chemistry. 2007; 282:5641-5652.

111. Mathew R, Kongara S, Beaudoin B, Karp CM, Bray K, Degenhardt K, Chen G, Jin S and White E. Autophagy suppresses tumor progression by limiting chromosomal instability. Genes \& development. 2007; 21:1367-1381.

112. Kimmelman AC. The dynamic nature of autophagy in cancer. Genes \& development. 2011; 25:1999-2010.

113. Aredia F, Guaman Ortiz LM, Giansanti V and Scovassi AI. Autophagy and cancer. Cells. 2012; 1:520-534.

114. Papandreou I, Lim AL, Laderoute K and Denko NC. Hypoxia signals autophagy in tumor cells via AMPK activity, independent of HIF-1, BNIP3, and BNIP3L. Cell death and differentiation. 2008; 15:1572-1581.

115. Kemp MG, Lindsey-Boltz LA and Sancar A. UV Light Potentiates STING (Stimulator of Interferon Genes)dependent Innate Immune Signaling through Deregulation of ULK1 (Unc51-like Kinase 1). The Journal of biological chemistry. 2015; 290:12184-12194.

116. Teplova I, Lozy F, Price S, Singh S, Barnard N, Cardiff RD, Birge RB and Karantza V. ATG proteins mediate efferocytosis and suppress inflammation in mammary involution. Autophagy. 2013; 9:459-475.

117. Takeshita F, Kobiyama K, Miyawaki A, Jounai N and Okuda K. The non-canonical role of Atg family members as suppressors of innate antiviral immune signaling. Autophagy. 2008; 4:67-69.

118. Hayes J, Peruzzi PP and Lawler S. MicroRNAs in cancer: biomarkers, functions and therapy. Trends in molecular medicine. 2014; 20:460-469.
119. Humphries B and Yang C. The microRNA-200 family: small molecules with novel roles in cancer development, progression and therapy. Oncotarget. 2015; 6:6472-6498. doi: 10.18632/oncotarget.3052.

120. Gibbings D, Mostowy S, Jay F, Schwab Y, Cossart P and Voinnet O. Selective autophagy degrades DICER and AGO2 and regulates miRNA activity. Nature cell biology. 2012; 14:1314-1321.

121. Zhang $\mathrm{P}$ and Zhang H. Autophagy modulates miRNAmediated gene silencing and selectively degrades AIN-1/ GW182 in C. elegans. EMBO reports. 2013; 14:568-576.

122. Korkmaz G, le Sage C, Tekirdag KA, Agami R and Gozuacik D. miR-376b controls starvation and mTOR inhibition-related autophagy by targeting ATG4C and BECN1. Autophagy. 2012; 8:165-176.

123. Guo X, Xue H, Guo X, Gao X, Xu S, Yan S, Han X, Li T, Shen J and Li G. MiR224-3p inhibits hypoxia-induced autophagy by targeting autophagy-related genes in human glioblastoma cells. Oncotarget. 2015; 6:41620-41637. doi: 10.18632/oncotarget.5871.

124. Ma Y, Yang HZ, Dong BJ, Zou HB, Zhou Y, Kong XM and Huang YR. Biphasic regulation of autophagy by miR-96 in prostate cancer cells under hypoxia. Oncotarget. 2014; 5:9169-9182. doi: 10.18632/oncotarget.2396.

125. Li D, Li X, Wang A, Meisgen F, Pivarcsi A, Sonkoly E, Stahle M and Landen NX. MicroRNA-31 Promotes Skin Wound Healing by Enhancing Keratinocyte Proliferation and Migration. The Journal of investigative dermatology. 2015; 135:1676-1685.

126. Rivetti di Val Cervo P, Lena AM, Nicoloso M, Rossi S, Mancini M, Zhou H, Saintigny G, Dellambra E, Odorisio T, Mahe C, Calin GA, Candi E and Melino G. p63-microRNA feedback in keratinocyte senescence. Proceedings of the National Academy of Sciences of the United States of America. 2012; 109:1133-1138.

127. Hildebrand J, Rutze M, Walz N, Gallinat S, Wenck H, Deppert W, Grundhoff A and Knott A. A comprehensive analysis of microRNA expression during human keratinocyte differentiation in vitro and in vivo. The Journal of investigative dermatology. 2011; 131:20-29.

128. Zhang Y, Liao Z, Zhang LJ and Xiao HT. The utility of chloroquine in cancer therapy. Current medical research and opinion. 2015; 31:1009-1013.

129. Poklepovic A and Gewirtz DA. Outcome of early clinical trials of the combination of hydroxychloroquine with chemotherapy in cancer. Autophagy. 2014; 10:1478-1480.

130. Griffin LM, Cicchini L, Pyeon D. Human papillomavirus infection is inhibited host autophagy in primary human keratinocytes. Virology. 2013; 437:12-19. 